\title{
Refractive index sensor with asymmetrical tapered fiber based on evanescent field sensing
}

\begin{abstract}
We report and demonstrate a single-mode asymmetric tapered fiber as a refractive index (RI) sensor based on evanescent field sensing. The proposed setup managed to sense the changes of RI when the tapered region was exposed to different concentrations of Sodium chloride $(\mathrm{NaCl})$. This was possible due to the output interference spectrum shifting in response to the changes of RI. Different environmental sensitivities were achieved by manipulating the transition lengths of the tapered fiber. Experimental results depict RI sensitivities of 3914.7 $\mathrm{nm} / \mathrm{RIU}$ (refractive index unit) and $3395.7 \mathrm{~nm} / \mathrm{RIU}$ for taper profiles with down-taper transition lengths of $2 \mathrm{~mm}$ and $8 \mathrm{~mm}$, respectively, which is higher than previously reported asymmetric taper RI sensors. Such findings may find itself beneficial in low cost, reliable and simplified sensors.
\end{abstract}

Keyword: Evanescent wave; Refractive index; Tapered fiber; Vytran 